\begin{tabular}{|l|l|l|l|}
\hline Eiszeitalter und Gegenwart & Band 17 & Seite 149-152 & Öhringen/Württ.,15.Dezember 1966 \\
\hline
\end{tabular}

\title{
Ein speläochronologischer Beitrag zur postglazialen Klimageschichte
}

\author{
Von Herbert W. Franke, Kreuzpullach \\ Mit 2 Abbildungen
}

$\mathrm{Z}$ us a m m e $\mathrm{f}$ as s u $\mathrm{ng}$. Höhlensinter bietet gute Voraussetzungen für die Anwendung der Radiocarbon-Datierung. Es werden einige Ergebnisse von Messungen an Stalagmiten mitgeteilt; die Untersuchung erfolgte im C14-Laboratorium des 2. Physikalischen Instituts der Universität Heidelberg. Nimmt man für sekundären Kalk einen Rezentstandard von $85 \%$ an, so liegen die Wachstumsperioden der untersuchten Stalagmiten innerhalb des postglazialen Klimaoptimums. Das bestärkt die Ansicht, daß die Wachstumsgrenzen von Sintergenerationen Zeitmarken für klimatische Ưbergangsphasen sind. Zur genaueren Prüfung der Situation ist eine Meßreihe geplant, aus der sich C14-Daten für die postglaziale Klimageschichte ergeben sollten.

$\mathrm{S} \mathrm{u} \mathrm{m} \mathrm{m} \mathrm{a} \mathrm{r} \mathrm{y.} \mathrm{Secondary} \mathrm{calcium} \mathrm{carbonate} \mathrm{sediments} \mathrm{in} \mathrm{caves} \mathrm{offer} \mathrm{good} \mathrm{presuppositions} \mathrm{for}$ the application of radio carbon dating. Some results of measurements on stalagmites are reported; they were received by the Cl4-Laboratory of the 2. Physikalisches Institut, University Heidelberg. If for secondary calcium carbonate a recent standard of $85 \%$ is assumed, the growth periods of the examined stalagmites lie within the postglazial climatic optimum. That strengthens the opinion that growth limits of sediment generations are time marks for phases of climatic changes. The situation will be examined in a planned series of measurements which should result in C14-dates for the postglacial history of climate.

Höhlensinter weist einige Eigenschaften auf, die ihn für chronologische Untersuchungen geeignet erscheinen lassen:

1. Als sekundärer Kalk enthält er das Kohlenstoffisotop $\mathrm{C}^{14}$ und ist daher der Radiocarbondatierung zugänglich (FrANKE 1951).

2. Die jüngsten Sintergenerationen stammen aus der Periode des Postglazials und liegen daher innerhalb der Meßgrenzen (Franke, Münnich \& Vogel 1961).

3. Höhlensinter ist seiner Natur nach vor den abbauenden Einflüssen der Atmosphärilien geschützt und vor Verlagerung weitgehend gesichert; eventuell erfolgte Bewegungen sind durch Sprungstellen zu erkennen.

4. Das Sintermaterial ist kompakt und Austauscheffekten viel weniger ausgesetzt als Knochen.

5. Höhlensinter ist geschichtet und läßt in seinen Folgen klimatische oder klimatisch bedingte Ereignisse erkennen, beziehungsweise ist mit solchen zu korrelieren (Franke \& Trimmel 1962).

Demgegenüber ist auch ein Nachteil zu erwähnen: Der Rezentstandard der sekundären Kalke bedarf noch einer genauen Bestimmung. Diese Unsicherheit vermindert die Meßgenauigkeit vorderhand um maximal eine Halbwertszeit, vermutlich aber um weniger.

Wie die Theorie zeigt, ist für die Bildung größerer Sintermengen Vegetation oberhalb der Fundstelle Voraussetzung (BöGLI 1954). Höhlensinter ist daher ein Kennmerkmal für ein dem Pflanzenwuchs zuträgliches Klima. Die starke, heute aktive Sinterentstehung in der mediterranen Klimazone kann als Beweis dafür gelten, und auch die ersten $\mathrm{C}^{14}$-Messungen von Höhlensinterproben bestätigen dieses Ergebnis. Es liegt daher nahe, die Basisund Deckflächen von Sinterlagen als Marken für die Anfangs- und Endzeiten der postglazialen Wärmestadien zu verwenden.

Diese Auffassung wurde zuletzt durch einige Messungen von D. Berdau und K. O. MüNNICH am C14-Laboratorium des Zweiten Physikalischen Instituts der Universität Heidelberg unterstützt. Als Proben dienten kerzenförmige Stalagmiten, die die Leitformen einer konstanten physikalisch-chemischen Situation sind und sich auch noch aus einigen anderen Gründen besonders gut für Datierungen eignen (FraNKE 1965). 
Ta be 11 e 1

I. Kalksinter eines Stalagmiten aus dem Katerloch bei Weiz, Steiermark, Ósterreich

\begin{tabular}{|c|c|c|}
\hline H $2268-1700$ & Katerloch A & \\
\hline H $2268-1701$ & Katerloch A & \\
\hline H $2268-1692$ & $\begin{array}{l}\text { Schnitt } 37 / 39 \\
\text { Katerloch C }\end{array}$ & 657 \\
\hline & Schnitt $0.5 / 1.5$ & $5760 \pm 75$ \\
\hline
\end{tabular}

II. Kalksinter eines Stalagmiten aus der Karls- und Bärenhöhle bei Erpfingen, Schwäbischer Jura, Deutschland
H 2269-1713
Bärenhöhle A
H $2269-1711$
Schnitt 42/44
Bärenhöhle C
Schnitt 3/5
$8150 \pm 110 \mathrm{~J}$.
$3660 \pm 65 \mathrm{~J}$

III. Kalksinter eines Stalagmiten aus der Alten Höhle (Prinzenhöhle) bei Hemer, Sauerland, Deutschland
H 2270-1729
Prinzenhöhle A
H 2270-1728
Schnitt 53/55
Prinzenhöhle D
Schnitt $1.5 / 4.5$
$5840 \pm 75 \mathrm{~J}$
$3870 \pm 80 \mathrm{~J}$

Anmerkung zu Tabelle 1: Die Angaben der linken Spalte sind die laufenden Nummern der vom Heidelberger C14-Institut gemessenen Proben. Die Schnittstellen unter- und oberhalb der scheibenförmig entnommenen Proben sind durch Zentimeterangaben, von der Basis aus gemessen, gekennzeichnet. Die Zeitwerte sind in Jahren vor 1950, dem Bezugsjahr der C14_ Altersdaten, angegeben, die Fehlergrenzen durch den mittleren Meßfehler. Als Rezentstandard des Kalksinters wurde $85 \%$ der Aktivität rezenter organischer Proben angenommen. Als Halbwertszeit diente der Wert 5568 Jahre.
Probe I
II
III
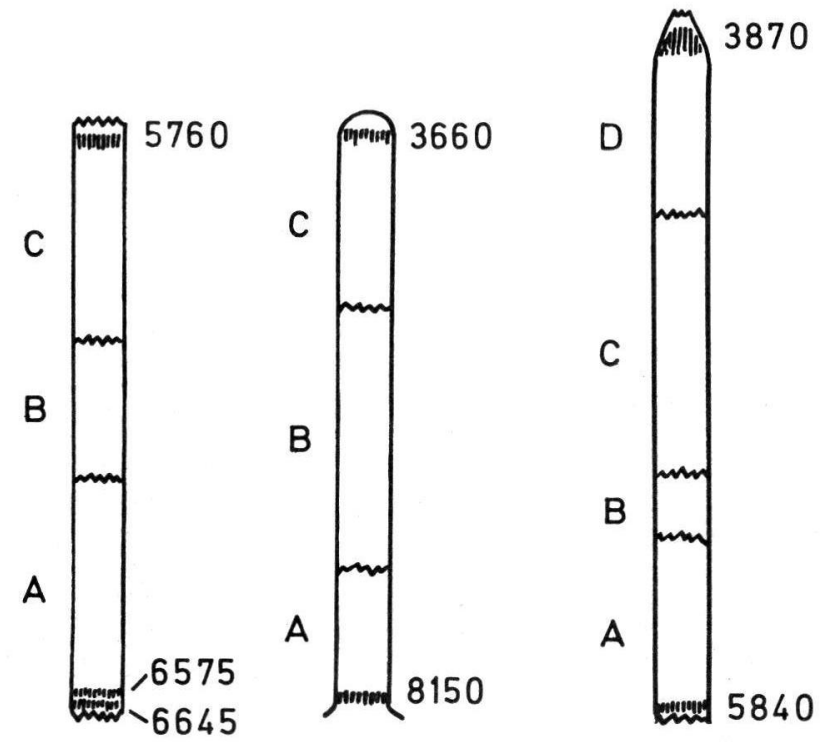

Abb. 1. Die Skizze gibt die zur Messung verwendeten Stalagmiten in ihren richtigen Längenverhältnissen wieder. Bruchflächen sind durch gezackte Linien angedeutet, die einzelnen Bruchstücke wurden mit A, B, C, D bezeichnet. Der Ort der Probeentnahme ist durch senkrechte Strichelung gekennzeichnet. Die Scheiben wurden geschält, um den Einfluß der aus jüngeren Abschnitten stammenden Randschichten auszuschalten. Bei den Jahresangaben (vor der Gegenwart) sind die Fehlergrenzen weggelassen. 
Die Messung hatte den Zweck, die Wachstumsgeschwindigkeit von Sinter festzustellen. Es kam also nur darauf an, gleichmäßig gewachsene Proben aus dem meßbaren Zeitbereich, also der jüngsten, gut meßbaren Sintergeneration, zu entnehmen. Die Auswertung ergab, daß alle aus dem warmfeuchten Atlantikum stammen, vorausgesetzt, daß der Rezentstandard richtig gewählt wurde. Diese Voraussetzung gilt auch für die folgenden Alterszuordnungen.

Die Deckabschnitte der Proben II und III haben dasselbe Alter - das heißt, daß sie trotz ihres verschiedenen Ursprungs ihre Wachstumsperiode zur gleichen Zeit beendet haben, und zwar während des Wechsels vom Atlantikum zum warmtrockenen Subboreal. Bei Probe I fehlt der Kopfabschnitt, so daß kein Vergleich mit ihr möglich war. In seiner vollen Erstreckung erhalten ist nur der Stalagmit II; er dokumentiert eine volle Wachstumsperiode. Sein Entstehungsbeginn liegt an der Grenze zwischen dem kühltrockenen Präboreal und dem warmtrockenen Boreal - in jenem Abschnitt, in den man das Ende der Eiszeit verlegt.

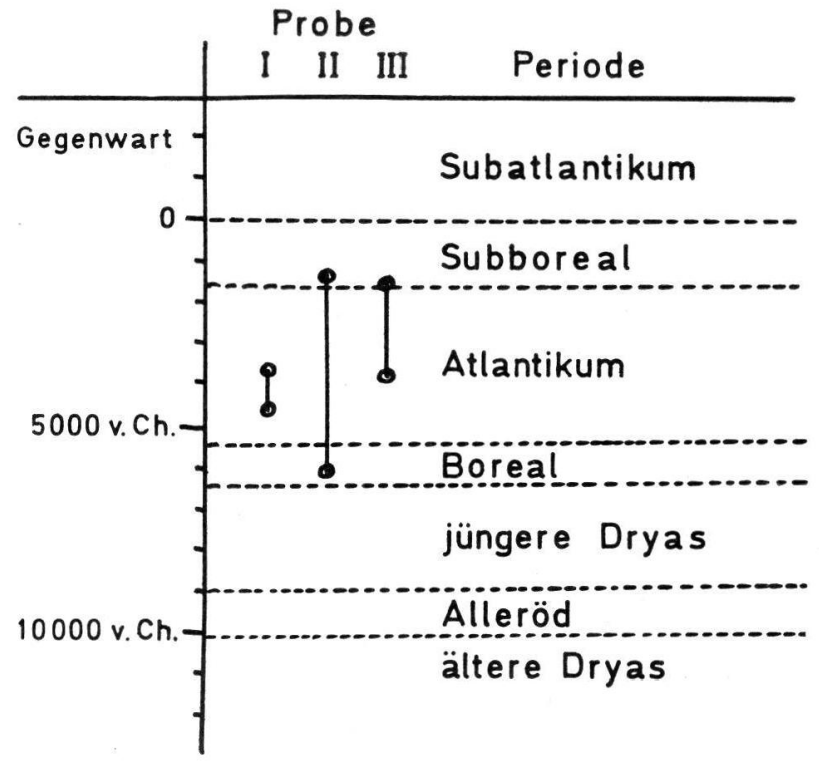

Abb. 2. Die Darstellung gibt die Einpassung der Tropfstein-Wachstumszeiten in den Kalender der postglazialen Klimaperioden wieder.

Schon bei diesen wenigen Stichproben zeigt sich eine deutliche Zuordnung der Wachstumsgrenzen zu klimatischen Übergängen. Es ist geplant, eine größere Serie von Sinterproben zu datieren, um aus der Statistik der Meßergebnisse neue Hinweise für eine genauere Einstufung der postglazialen Klimageschichte zu gewinnen.

Die Radiocarbonanalysen wurden im C14-Laboratorium des Zweiten Physikalischen Instituts der Universität Heidelberg vorgenommen, wofür ich den Herren D. BerdaU und Dr.K.O. MüNNICH zu besonderem Dank verpflichtet bin. Weiter habe ich den Herren H. Hofer, Dürntal, Steiermark, K. Bez, Erpfingen, Schwaben, und K.-H. Pielsticker, Hemer-Sundwig, Sauerland, für die Beistellung der Sinterproben zu danken. 


\section{Literatur}

FrANKE, H. W.: Altersbestimmung von Kalzitkonkretionen mit radioaktivem Kohlenstoff. Naturwissenschaften 38, 527, 1951.

Franke, H. W., MünNICH, K. O., Vogel, J. C.: Die Radiokohlenstoffdatierung von Kalkabscheidungen. Atompraxis 7, 298, 1961.

Franke, H. W., Trimmel, H.: Radiokarbondatierungen an Sinterproben der Griffener Tropfsteinhöhle. Carinthia II, 152, 108, 1962.

BöGli, A.: Das Verhalten von Karbonaten in der Natur. Die Höhle, 5, 36, 1954.

Franke, H. W.: The Theory behind Stalagmite Shapes. Studies in Speleology 1, 89, 1965.

Manuskr. eingeg. 18. 7. 1966

Anschrift des Verf.: Dr. H. W. Franke, 8024 Kreuzpullach, Post Deisenhofen, Bayern. 